\title{
Efficiency of Fuzzy C Means algorithm for Brain Tumor segmentation in MR Brain Images
}

\author{
T.Velmurugan ${ }^{1}$ S. Mahalakshmi ${ }^{2}$ \\ ${ }^{1}$ Associate Professor, ${ }^{2}$ Research Scholar, \\ PG and Research Department of Computer Science, D. G. Vaishnav College,Chennai-600106, India. \\ E-Mail: 1'velmurugan_dgvc@yahoo.co.in,2'smaha25@gmail.com
}

\begin{abstract}
Background and Objective: Image processing is a technique or set of operations to get meaningful information from an image for the usefulness and effectiveness of images. Image segmentation is an efficient technique in extracting and separating some of the features in the images. Methods: The main objective of this research work is to find the best fit of FCM algorithm over finding the axial and coronal plane of MRI brain imagesvia its accuracy and computational time.In the preprocessing, brain images of MRI have been converted from the DICOM format into standard image. Preprocessing is carried out by Gaussian filter technique to remove the noises in the images. The Fuzzy $\mathrm{C}$ Means (FCM) algorithm is implemented to segment the tumor affected region in the MR images. Results: By comparing the histogram values of the images (before and after segmentation) with the cluster center values by the FCM algorithm, the efficiency and accuracy of the algorithm is evaluated. Conclusion: The best fit of FCM algorithm into the axial and coronal plane is identified based on the computational time in this work.
\end{abstract}

Keywords: Brain tumor, image segmentation, Fuzzy C Means algorithm, Magnetic Resonance Image.

\section{Introduction}

Image processing is a new methodology which converts image into a digital form and perform some operation on it in order to get an enhanced image or to extract some meaningful and useful information from it. Image processing is basically of two aspects: one is improving the visual appearance of images to a human viewer and the secondmeasuring some of the features and structures present in the images. The digital image can be optimized for the application by segmenting or altering the appearance of structures within it based on the body part, diagnostic task, viewing preferences etc. Analyzing the images in the computer will help the radiologists and to detect the important or suspicious region in the image to diagnose the disease.

An exciting source of images is the medical field. Imaging plays a unique importance practically in the medical informatics. The field of biomedical analysis is rapidly evolved with the last two decades. Over the years crossed, the major technical challenge in the images is increase in size and its dimensionality. In medical scenario, we have so many algorithms which automatically or semi-automatically detect lesion, diseases and tumors and enhance some of the locations in the images. The research process of medical image analysis has two dimensions one with the patient-centered medical world and the second one is computer-centered medical world.There different imaging modalities such as CT-Computed Tomography, PET-Positron Emission Tomography, MRI-Magnetic Resonance Imaging, Ultrasound and X-Ray[15]. The efforts of the research in the medical field is mainly devoted on processing and analyzing medical image to segment the meaningful data such as shape, to detect abnormalities, quantifying the changes in the position and growth of the suspected regions, motion of organs and its volume.

In the medical domain the MRI evolution creates a huge revolution. In 1971, Prof. Raymond Damadianinitials the first concept of MRI. From the day of invention, researchers and doctors keep on improvising and using the MRI scans not only to assist in medical diagnose but also in the research area. An MRI scanner is an important tool to analyze patients and produces the MRI images. The scanner is developed with two powerful magnets, the first magnet aligns the water molecules in one direction either north or south and second one is to generate the quick pulses series in turn on and off.The magnets should be cooled constantly in the temperature of absolute zero $\left(-459.67^{\circ} \mathrm{F}\right)$. By traditionally liquid helium is act as cooling agents for the magnets.The MRI scan images are useful to predict uterine abnormalities in women for the treatment of infertility, some types of heart problems, injuries and abnormal behaviors of bone joints, detect the lumps in the liver and abdominal organs, abnormalities in the brain and spinal cord, pelvic pain for women and etc.

Image segmentation is the process of partitioning an input image into set of connected pixels. Technically, image segmentation is refers to the decomposition of a scene into different meaningful regions. Scientifically, the image segmentation is a vision task of hypothetical middle-level. The aim of the image segmentation is to identify the region of interest i.e., to locate tumor and radiation dose calculation in radiation 
therapy in automatic segmentation of medical images [8]. The segmentation is based on the measurements taken from the images based on the texture, color, depth, motion or intensity values of an image. In general, image segmentation is a step by step process to study all the image regions in depth. One of the major applications of image segmentation is to identify the objects in the scene and measuring its shape and size. In this research work, the MRI brain images are analyzed with the FCM algorithm and the results are verified with the histograms values of the images to evaluate the accuracy of the results. This article is organized as follows.Section II deals with the literature review of the variousrelated works, section III discusses about the materials and methods applied in this research work.The results and its discussion are explained in section IV and finally section $\mathrm{V}$ concludes the research work.

\section{Literature Survey}

There are many researchers performing research to find the efficiency of tumor affected region to help the radiologists. In particularly, MRI brain image the performance of the FCM is highly important clustering algorithm for its efficiency and effectiveness. Some of the research works for different persons are discussed here from which gives a different perspective of FCM. 3D brain tumor segmentation in MRI using fuzzy classification, symmetry analysis and spatially constrained deformable models discussed about the segmentation of brain tumor. Fuzzy classification and approximate brain asymmetry plane based on these two different approaches the tumor detection is carried out and its effective for all different types of tumor[10]. M.N. Ahmed etal. are carried out in their research work in bias field estimation and adaptive segmentation of MRI data using a modified fuzzy c-means algorithm. They proposes a novel algorithm to segment the MRI data and evaluate the inhomogeneities intensity of fuzzy logic[1]. Current Methods in the Automatic Tissue Segmentation of 3D Magnetic Resonance Brain Images they provide the current methods in the tissue segmentation with the detailed study of each method with the mathematical representation and advantages and disadvantages of the methods. They provide the conventional fuzzy c-means with the two ideas intensity nonuniformity INU and spatial context with the pixel values in clustering process [12].

Automatic Tumor Segmentation Using Knowledge-Based Techniques is research work done by Matthew C. Clarkand etal. The suspected tumor is identified by multispectral histogram analysis and region analysis is used for the intracranial region. They generated a system that automatically identifies tumor segments and labels of glioblastoma-multiforme tumors in the human brain with the help of magnetic resonance images [6]. Discrete dynamic contour model with adjacent vertices consists of vertices and edges is the main evaluation criteria to segment thalamus from MRI brain images which is an important neuro-anatomic structure in brain is the research work discussed by LadanAmini and et.al [4]. Intensity space map(ISM) is combined with the fuzzy c-means clustering algorithm to segment the color MRI images for tumor detection work. Though the manual segmentation of MRI data is possible, this algorithm segments the muscle regions and its time consuming[19].

Keh-Shih Chuang, Hong-Long Tzeng, Sharon Chen, Jay Wu and Tzong-Jer Chen carried their research work in Fuzzy c-means clustering with spatial information for image segmentation. The spatial information incorporates with the fuzzy c-means algorithm for clustering into the membership function. Each pixel is taken consideration with the summation of spatial function with the neighborhood membership function[5]. Another research work proposes a new automatic clustering approach with a hybrid algorithm in the combination of Artificial Bee colony with fuzzy c-means to determine the tumor region. The hybridization of (FCMAB), to segment the MRI brain image extracts abnormal cell growth with the cluster centers [3]. The log bias field is stack of spline surfaces, that reduces the spline coefficients of 3-bias field reduces in finding the spline coefficients is effective two-stage algorithm. They proposed algorithm inorder to account the spatial continuity constraints between image volume element. The MR imaging signal is formulated by a multiplicative bias field with INU artifact [11].A Modified Fuzzy C-Means Algorithm for Bias Field Estimation and Segmentation of MRI Data is a research work carried by Mohamed N. Ahmed. The objectivefunctions of the standard fuzzy cmeans algorithm for inhomogeneities and allow voxel the volume element of an image is influences the adjacent neighborhood. Theregularize and biases of the neighborhood pixels leads towards the homogeneous labeling in the piecewise information [2].

Brain Tumor Detection Using MRI Images is done by the research authors of PranitaBalaji Kanade1 and P.P Gumaste. They proposed algorithm which consists of six stage process which includes test images,preprocessing, denoising and SWT, segmentation, feature extraction and SVM/PNN. The algorithm is have higher accuracy and low error rates. The image segmentation algorithm will have following features accuracy, reliability, repeatability, robustness and least dependency[8]. Ming Zhao and etal.carried their research work titled as Automatic Threshold Level Set Model Applied on MRI Image Segmentation of Brain Tissue [20]. A mathematical representation and proof for Chan and Vese model for the different mean and variance for automatic threshold level set for the image segmentation. The extraction of MR images was developed by threshold level set without edges for the tissues in brain.Dzung L. Pham and Jerry L. Prince evaluated a novel algorithm with the multiplicative intensity inhomogeneities for the obtaining the fuzzy segmentation of images. An iterative algorithm is developed to minimize the objective function of fuzzy c-means [13]. 


\section{Materials and Methods}

Dunn was the first person to introduce the Fuzzy C-Means clustering algorithm and it's extended by Bezdek.Duun Clustering is a widely used technique to classify images, that pixels of same group are belongs to one group and differentiated pixels are belongs to some other groups [15].The MRI scans has been taken for the patients for many reasons. To detect and identify bleeding, injury, blood vessels, tumors in brain area. MRI also diagnoses more problem than on X-ray, ultrasound scan or CT scan. Brain is the main central nervous unit which connects all the nerves of the body. It's very important for the patients to diagnose the various problems in the brain. So the detection and prediction of the brain tumor affected region is must and it should be precise for the radiologists to produce accurate results to the physicians. Fuzzy c-means (FCM) clustering algorithm is a commonly used in image segmentation, to assigns pixels of the images into distinct classes based on the features of the image. One of the important domains of Fuzzy C Means is medical image analysis. This paper analysis and detect brain tumor affected region separately with the help of the FCM in the coordination of histogram values of the image. The MRI scanner scans the brain and produces the resultant image in three different planes for the detailed description. The magnetic resonance scanner provides various scanning images of the brain from different directions. Figure 1 shows the different planes of the MRI brain images. One is axial plane which slices the brain and provides the information, the second one is the coronal plane shows the information from the back with the spinal cord and the last one is the sagittal plane. The sagittal plane describes the brain image from the left and right side of the brain anatomy shows in the Fig. 1.

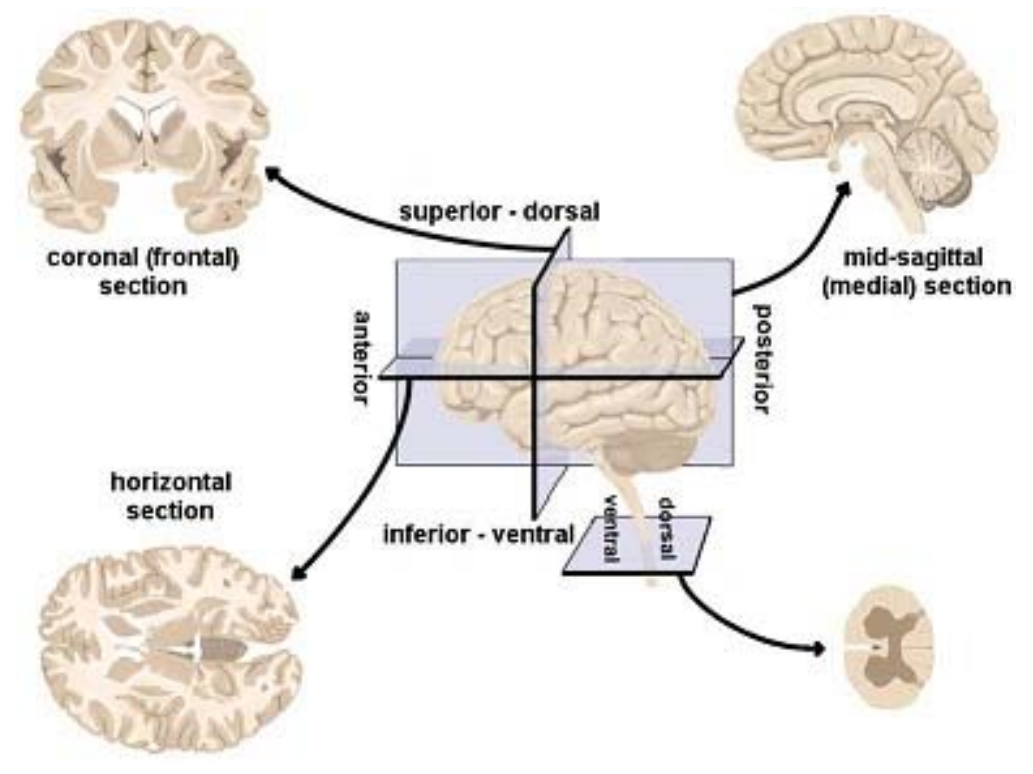

Fig.1. Different planes of MRI brain image.

The FCM clustering algorithm is an iterative process to produce the cluster centers partition by minimizing the weighted group of squared error objective functions summation. The MRI brain image is segmented by FCM algorithm is carried by many researchers. In this paper, the segmented region pixel values is verified with the histogram of the resultant images. The histogram values of the resultant image is been analyzed. The research methodology of this research work includes four modules. The first module is converting the DICOM images in to some real world images which can be easily handled by any software. The second module is preprocessing the input image by removing noise. The third module is applying the traditional FCM algorithm to the preprocessed images and finding the cluster values to the resultant images and the last module is generation of histogram values and cross examining with the results from the third module to find the accuracy with the coordination of pixels intensity. The resultant images produce the segmented region of the input MRI brain images based on the evaluation of the intensity values of the images. By segmenting the image the cluster center values are been recorded with the help of FCM algorithm.

\subsection{Gaussian filter}

The MRI input brain imagenoises areremoved with the Gaussian filter in the preprocessing stage. The Gaussian noise is an form of white noise. It is caused due to the signals random fluctuations. The white noise is a random signal with spectral densities flat power and it is also called as additive noise [9]. An simple and important filtering techniques for images is the Gaussian filtering which is a subtitle of bilateral filtering. In the Gaussian filtering function both the closeness function and similarity function are the Gaussian function 
arguments of the Euclidean distance. The closeness function is described in the equation 5 and the similarity function is illustrated in the equation 6 [14].

$$
c(\xi, x)=e^{-\frac{1}{2}\left(\frac{d(\xi, x)}{\sigma_{d}}\right)^{2}}
$$

where $\mathrm{d}(\xi, \mathrm{x})=\mathrm{d}(\xi-\mathrm{x})=\|\xi-x\|$ is the Euclidean distance between $\xi$ and $\mathrm{x}$.

$$
s(\xi, x)=e^{-\frac{1}{2}\left(\frac{\delta(f(\xi), f(x))}{\sigma_{d}}\right)^{2}}
$$

Where $(\emptyset, f)=\boldsymbol{\delta}(\emptyset-f)=\|\varnothing-f\|$ is a suitable measure of distance between the intensity values of $\emptyset$ and $\mathrm{f}$. The geometric spread $\sigma_{\mathrm{d}}$ is the domain is chosen based on the desired amount of low-pass filtering. The Gaussian range filter is insensitive to the overall filters and additives changes are subjected to image intensity.

\subsection{The Fuzzy C-Means Algorithm}

Fuzzy c-means is clustering algorithm to classify the pixels into two or more groups. FCM is mainly used to segment the images. Though, several approaches are exists in the real world for MRI brain image the FCM produces more efficient and effective results than the others. The FCM clustering algorithm works on the image based on some features like intensity values, texture, and pixels regions etc. In this approach, we cluster the image by taking the intensity of the pixels. The merely same intensity values of the pixels will belongs to one cluster and other pixels which may have the same intensity values form the next cluster. FCM clustering algorithm is based on minimizing the weighted square mean error of the objective function

$$
Y_{m}=\sum_{i=1}^{c} \sum u_{i j}^{m}\left\|X_{i}-C_{j}\right\|^{2}
$$

The above equation is the weighting function of the FCM clustering algorithm. Centroids cluster of I is in $C_{i}$ and the $u$ value is the range of between 0 and 1 ; Euclidean distance between the $i^{\text {th }}$ centroids and $j^{\text {th }}$ data joint is represented by $\mathrm{d}_{\mathrm{ij}}$ and weighting function is $\mathrm{m} £(1, \infty)$ [7].

Objective function of FCM

The procedure convergesthe saddle point of $Y_{m}$ or minimizes the local minima. The fuzzy segmentation of image data is done by the iterative process by optimizing the objective function is carried as follows

1. $\mathrm{C}$ and $\mathrm{q}$ values are to be set.

2. Initialize the matrix $\mathrm{M}=\mathrm{M}\left(\mathrm{M}_{\mathrm{ij}}\right)$

3. Loop counter $b=0$.

4. Center vectors calculation is done by $R^{k}=\left[R_{j}\right]$

$$
R_{i j}=\frac{\sum_{j=1}^{n} M_{i j}^{m} x_{i}}{\sum_{j=1}^{n} M_{i j}^{m}}
$$

5. $u^{(k)}$ and $u^{(k+1)}$ are to updated.

$$
u_{i j}=\frac{1}{\sum_{k=1}^{c}\left(\frac{\left\|X_{i}-c_{j}\right\|}{\left\|x_{i}-c_{k}\right\|}\right)^{2 /(m-1)}}
$$

6. $\max _{i j}\left\|M^{k+1}=M^{k}\right\|<\in$ is the termination condition

This iteration process is stop when it meets the following condition. The termination criterion with the range between 0 and 1 , and $\mathrm{k}$ is the number of iteration steps. In the above equations $\mathrm{m}>1$ and $\mathrm{x}_{\mathrm{i}}$ is data measured in d-dimensional, $M_{\mathrm{ij}}$ is the $\mathrm{X}$ membership degree in the $\mathrm{j}$ cluster and center of the cluster is in the $\mathrm{d}$ dimension of $\mathrm{R}_{\mathrm{j}}[7]$. The $\|*\|$ is expressing the similarity between center and the measured data. The optimization of the objective function is carried out in the segmentation of fuzzy, with the update of the cluster centers and objective functions of the equation(1) [17].The above equations 2 and 3 are the FCM to create clusters of the MRI brain image to segment and separated the tumor affected region based on the intensity values of the image.In the, FCM the $\mathrm{C}$ represents the clustering which means creating group of objects which are similar belongs to one group and dissimilar are belongs to different group. 


\section{Application Areas of FCM}

The applications of clustering techniques are document categorization, customer/market segmentation, scientific data analysis, city planning, land use and in earthquake studies [16]. Fuzzy, hard, remote sensing, satellite signal receiving, are the broad classification area of clustering algorithms. The clustering is widely used in many research areas such as data mining, artificial intelligence, fuzzy systems, pattern recognition, machine learning etc. The application of cluster analysis is widely used in chemistry, the systematize chemical and physical properties are analyzed and give detailed report in the field of analytical chemistry. The FCM is been applied in the data mining domain widely. The comparative analysis of FCM with $\mathrm{k}$ Medoids for the statistical data points are analysis in the research work and the results are discussed. The experimental results are discussed and effectiveness of the FCM is shown clearly for the distributed data points [18].

3.3 Histograms

Histogram is a graphical representation of an image based on the intensity distribution of an image and quantifies the each intensity values based on the number of pixels considered. In this work the detection of affected region is identified based on the intensity level of images using FCM [10]. If $\mathrm{f}$ be a image it is been represented by $\mathrm{m}_{\mathrm{r}}$ and $\mathrm{m}_{\mathrm{c}}$ matrix of pixel integer intensities ranging from 0 to L-1. The set of possible intensity value is 0 to 255 .

$$
p_{n}=(\text { number of pixels with the intensity } \mathrm{n}) /(\text { total number of pixels })
$$

The value of $\mathrm{n}$ is starts from 0 to L-1. To obtain the appropriate segmented results and to cross-checking the FCM we coordinate the histogram values with the cluster centers of the FCM.

\subsection{Proposed Method}

The main goal of this research work is to diagnose and detect the tumor affected region of brain with the Fuzzy c-means clustering algorithm. The structured methodology for this research work is discussed as follows:

Step1: In the MRI scan, the imaging results are the DICOM (Digital Imaging and Communication in Medicine) image format which is unreadable format by many software. The imagesare converted into standard image file formats .jpg or .gif. Since, the conversion of the images is necessary for further analysis.

Step 2: The preprocessing is done by removing the noise with the Gaussian filter to remove the white noise. Since, the clustering is based on the pixels values.

Step 3: Implementing the traditional fuzzy c-means algorithm to segment the images and to detect the tumor affected region of the axial and coronal side of the MRI brain slices.

Step 4: Generating the histograms values before and after for the images and record the computational time. Since comparing the histogram values with the cluster center values of the images.

The MRI brain image taken in this research work is real time data collected from Swami Vivekananda Diagnostic Center scan center in Chennai. Different patient's data from different age and places are taken and the proposed approach has been analyzed. The MRI brain images has three different planes in this work we have taken only two planes axial and coronal plane and comparing the performance of each plane for the FCM. The research work is to find the best fit of the FCM algorithm over the axial and coronal plane of MRI brain slices based on the computational time. Since, timely detection and prediction of any diseases is a highly critical area in all the diagnosis. However, the time taken for an algorithm to complete its evaluation is also important in the resource utilization.

The sample dataset consists of 40 MR images 20 from axial plane and 20 from coronal plane are taken to detect the tumor affected region. The sample images are 3D truecolor images i.e. the intensity range is between 0 and 255. To implement the FCM algorithm for the sample images noise removal plays an important role. Since, the segmentation is based on the intensity values of the images; the noise removal improves the quality of the image. The image noises are removed with the help of the Gaussian filter. The Gaussian filter is the simple and important technique for images to remove the white from the images. Since, the tumor affected region is been highlighted by white color in the image, so the removal of white noise helps a lot for the further implementation of images. The preprocessed images are undergone with the traditional objective function of FCM algorithm and results with the tumor affected region in the coordination of cluster center values. The histogram values of before and after segmentation with the FCM of the images are analyzed.

\section{Results and Discussion}

The main aim of this research work is to detect the tumor affected region separately from the MRI brain images and finding the best suitability of the algorithm in order to find axial and coronal plane extraction. The comparative analysis of FCM algorithm for the axial and coronal plane is carried out based on the computational time and its efficiency. Everything is possible with the images with the help of human vision. But, we may not sure, that all the radiologists have good vision and always detect the tumor correctly. Computerization of some of the practices in the medical area will help them to diagnose the disease in fast and effective manner. The FCM algorithm is one of technique to detect the tumor affected region separately from the brain for further studies. The best suitability of the FCM algorithm over the axial plane and coronal plane are calculated based on 
the computational time and the visibility of the images. This research work, the images of the brain are taken and analysis, it with the help of FCM algorithm. The results are cross examined with the histograms values to maintain the accuracy of the results. The first step is to convert the DICOM file format of the MRI brain image into some standard image file format. In this work, we converted the entire file in .jpg format. The images of the MRI brain are in three different planes. The axial transversal plane gives the slices of the brain in detail, from which we can see the center and interior part of the brain. The coronal plane show the back side of brain with the some spinal cord region and the last one is sagittal plane describes the left and right side of the brain in detail. In this study, we worked on the axial plane and coronal plane image in order to find, which plane the FCM algorithm is more suitable based on the computational time. As an example 3 images from both the axial and coronal plane are taken and Fig. 2 shows three sample images of the axial plane.

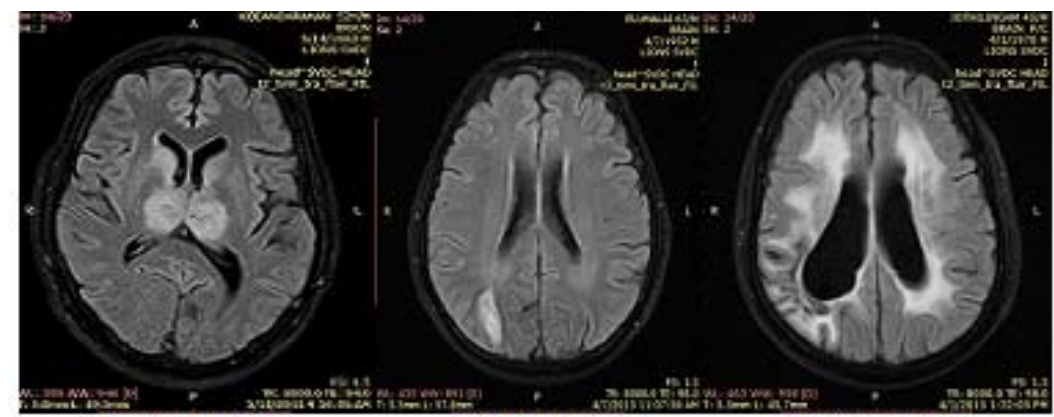

$\begin{array}{lll}\text { (a) AXI_IMG_01 } & \text { (b) AXI_IMG_02 } & \text { (C) AXI_IMG_03 }\end{array}$

Fig.2.Input abnormal images of the axial plane.

The second step is preprocessing starts with 3 MRI image for three different persons AXI_IMG_01 by removing the noise. The noises in the images are removed with the gaussian filtering techniques. Some of the pixels could be affected some form of white or some other noise so the removal of noise by gaussian technique will produce more standard resultant image. Since, the MRI images are black and white the gaussian filterin gtechnique might be correct choice inorder to get a clartity of the image. The results of the preprocessing module is shown in the Fig. 3. The third step is implementing the fuzzy c-means algorithm with the steps are evaluated as in the objective function of FCM. The equations of the FCM are implemented IBM machine with intel®core@ Duo processor and 8 GB RAM, running Windows7 operating system. The algorithm was developed via Matlab (R2008a). In this work, the segmentation of images is based on the intensity values of pixels in the imaging plane. The FCM is a clustering algorithm which gives the resultant images or values based on the cluster center of the pixels. The default segmentation level of the FCM algorithm set as $n=2$ are shown in the Fig. 3(b) and 3(c). The images are been segmented based on the intensity values of pixels; however the cluster center produces the highest point of intensity values of the image. The last level, separate the brain tumour affected region shown in the Fig. 3(d).

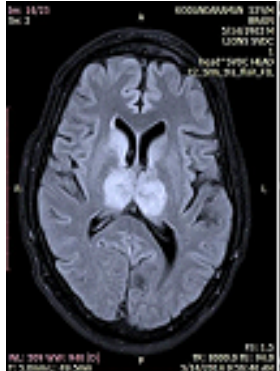

(a)

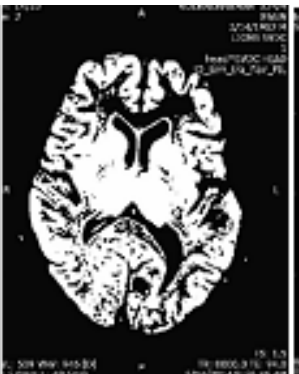

(b)

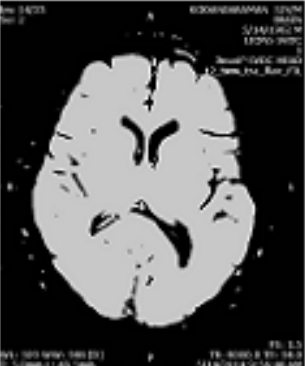

(c)

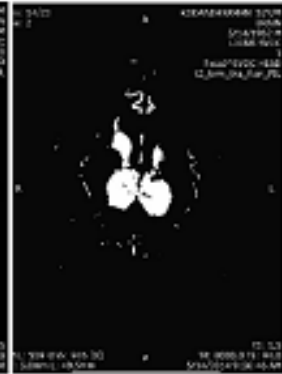

(d)

Fig.3. Preprocessing and FCM result of AXI_IMG_01.

The last step in this research work is cross-examing the cluster centers of FCM algorithm with the intesnity values of the resultant image with the histogram graph. The diagnoziation of brain tumor is highly important. We are segmenting the MRI brain images based on the pixels intensity values, so on reexaming the resultant values with the histogram graph give more adequate results. The resultant image of the FCM is compared with the histogram values of the image before and after segmentation. Since, the segmentation is based on the intensity values, the cluster values gives the intensity range of the image. With this, the histogram graph are been compared to achieve the correctness of the image. the validation of the resultant image is been 
analysed with the histogram graph vlaues for more accuracy. The cluster centers of the AXI_IMG_01 are $\mathrm{c} 1=101.2571$ and $\mathrm{c} 2=6.6779$.

The cluster center values obtained in the FCM algorithm is matches with the histogram graph values which shows its efficiency and correctness of the algorithm. The segmentation of images through the FCM algorithm is achieved by the intensity values of the images. The FCM algorithm segments the images and produces the cluster center values of the images and resultant images. However, in the first level of segmentation the cluster center values produces the highest intensity values of the images and in the next level it again segments the image and produces the cluster center values.After receiving the resultant images the histogram graph for both the image i.e. before and after segmentation is been analyzed with the cluster center values of the images. The segmentation is based on the intensity values of the pixels it has been cross examined with the histogram graph of the images. Fig. 4(a) shows the histogram value of AXI_IMG_01 before FCM, and its value is peaks at 100 which are same as the cluster center $\mathrm{c} 1$ of the image in the $\mathrm{FCM}$ clustering algorithm and the Fig. 4(b)depicts the tumor affected region separately with the same histogram values as with cluster center c2 of the AXI_IMG_01.

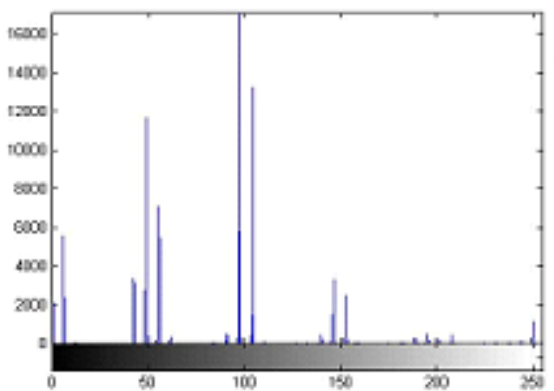

(a)- Before FCM

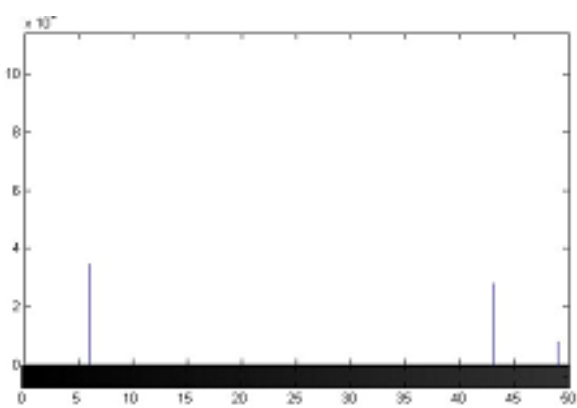

(b)- After FCM

Fig.4.Histograms of FCM forAXI_IMG_01

The axial plane image AXI_IMG_02 sample data is implemented as same as the AXI_IMG_01. The FCM clustering algorithm produces the same result as with the coordination of histogram. The cluster center values of AXI_IMG_02 is $\mathrm{c} 1=109.0320$ and $\mathrm{c} 2=10.330$. Fig. 5 shows the preprocessing and various steps of AXI_IMG_02 and separated brain tumor affected region separately. Cluster center values are compared with the histogram graph intensity of the AXI_IMG_02 before and after segmentation are shown in the Fig. 6. The process is implemented for AXI_IMG_03 with the cluster centers $\mathrm{c} 1=128.5591$ and $\mathrm{c} 2=13.4650$ and the results are shown in the Fig. 7. andFig. $\overline{8}$.
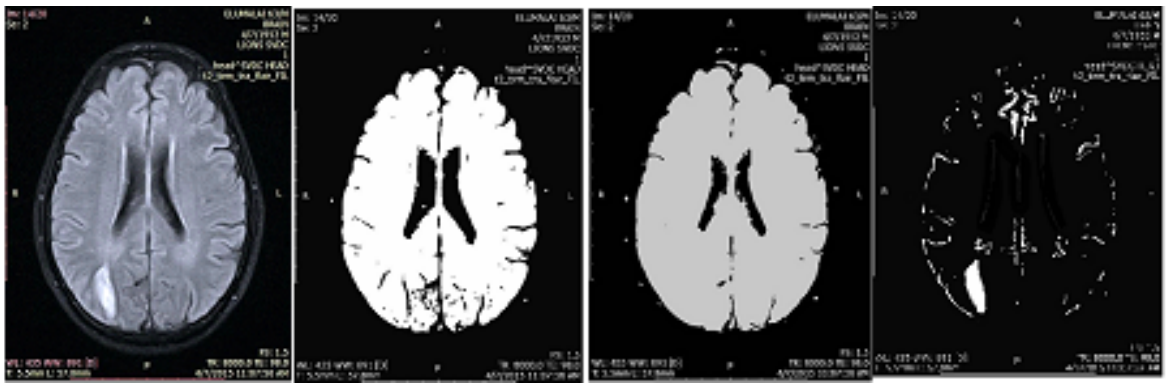

Fig.5. Preprocessing and FCM results of AXI_IMG_02.

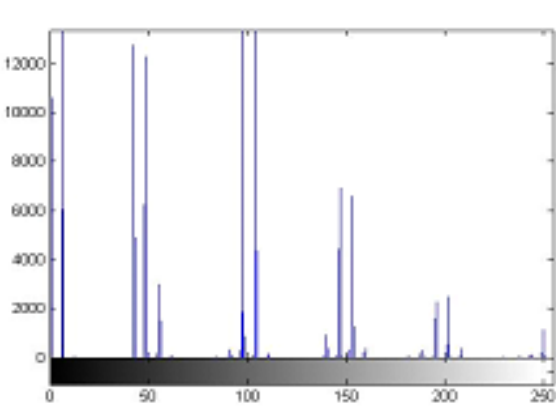

(a)- Before FCM

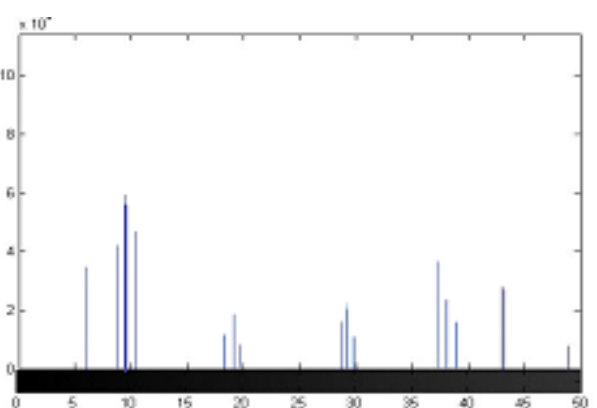

(b)- After FCM

Fig.6. Histograms of AXI_IMG_02 

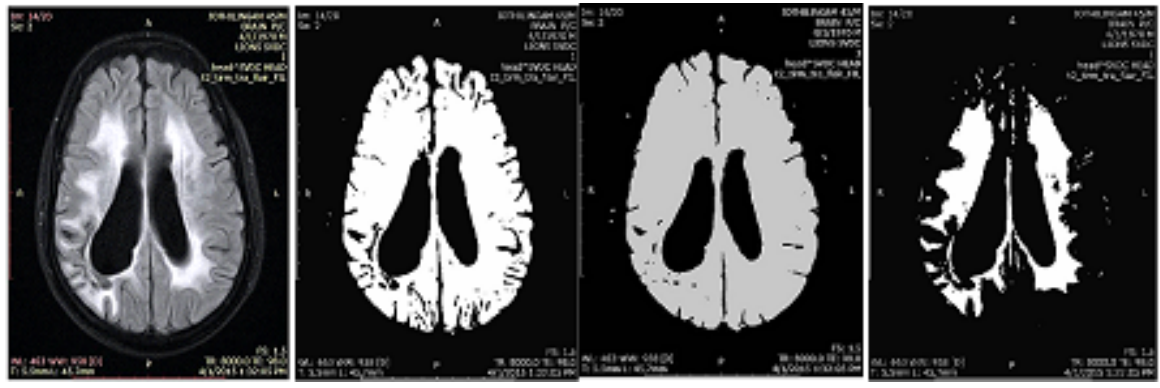

Fig.7. Preprocessing and FCM results of AXI_IMG_03.

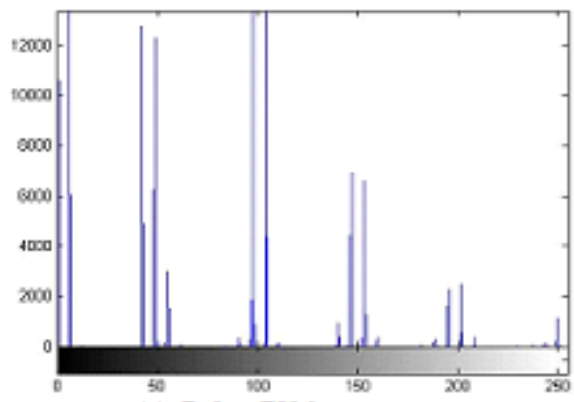

(a)- Before FCM

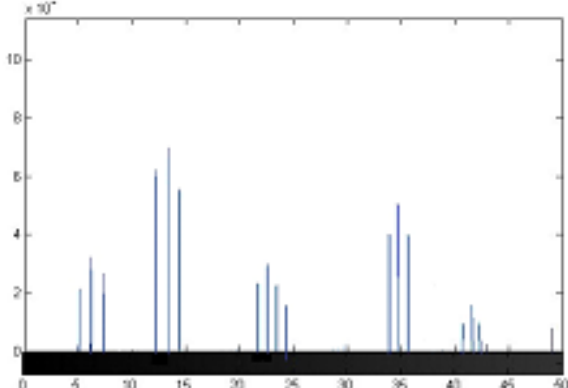

(b)- After FCM

Fig.8. Histograms of AXI_IMG_03.

Table I: Computational time of the axial plane.

\begin{tabular}{|llll|}
\hline MRI IMAGE DATA SET & CLUSTER CENTER - C1 & CLUSTER CENTER - C2 & RUN TIME (sec) \\
AXI_IMG_01 & 101.2571 & 6.6779 & 40.492280 \\
AXI_IMG_02 & 109.0320 & 10.330 & 26.659561 \\
AXI_IMG_03 & 128.5591 & 13.4650 & 39.257596 \\
\hline
\end{tabular}

The table I shows the computation time values of AXI_IMG_01, AXI_IMG_02 and AXI_IMG_03. The computation time is the CPU execution time of FCM algorithm which not includes the preprocessing time. The computation time is calculated in seconds. Fig. 4(a) shows before segmentation with the full intensity value is peak at the somewhere between 120 and 130. The value is equal to cluster center $\mathrm{c} 1=128.559$. After the segmentation the histogram value is peak at the point 10. Fig. 4(b) segmented region intensity value is equal to the cluster center value $\mathrm{c} 2=10.330$ of FCM algorithm which shows the accuracy of the segmented region.

The cluster centers of $\mathrm{c} 1$ and $\mathrm{c} 2$ of the sample data are shown in the table. The table shows the cluster center values of the image before and after segmentation of images. This value is approximately equal to the histogram peak point values for the corresponding images to maintain the accuracy and to achieve adequate results. Diagnosing tumor plays an important role in patients to produce accurate results is a highly an important criteria. The coronal plane is another plane in the MRI brain image which shows some tumor affected region with the back plane of the human. In the axial plane we can detect some types of tumor. The coronal plane sample MRI data set is shown in the Fig. 9. COR_IMG_01 data is also preprocessed with the Gaussian filter technique. The cluster values of COR_IMG_02 is $\mathrm{c} \overline{1}=40 . \overline{0} 859$ and $\mathrm{c} 2=182.6475$ and the results are shown in the Fig. 10.and histogram graph of the image are shown in the Fig. 11.

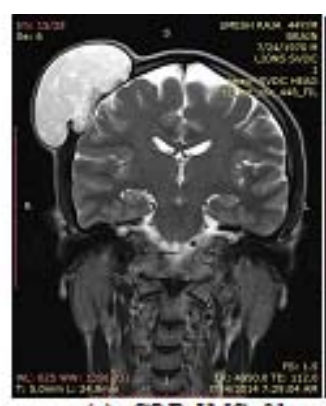

(a) COR_IMG_01

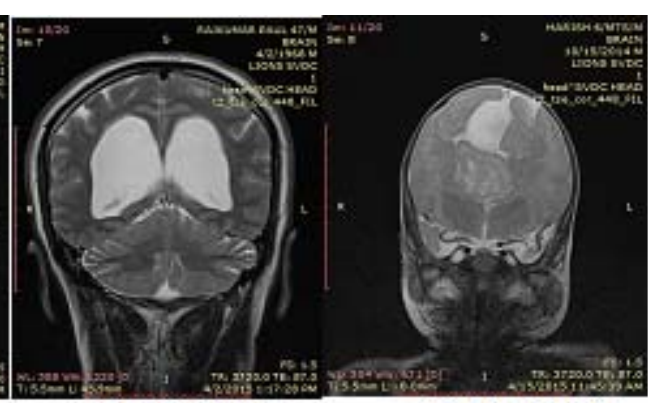

(b) COR_IMG_02 (c) COR_IMG_03

Fig.9. Input abnormal images of the coronal plane. 


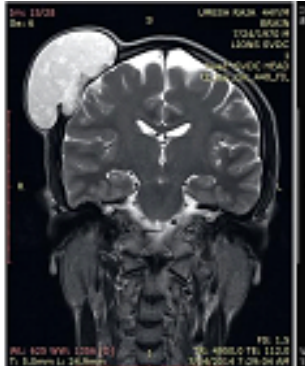

(a)

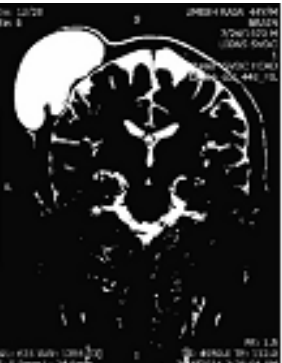

(b)

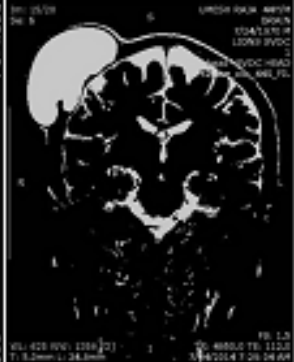

(c)

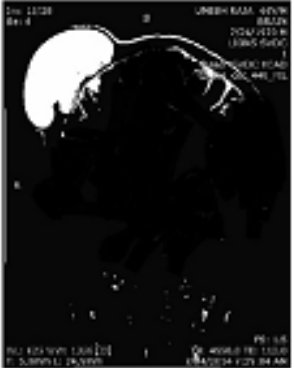

(d)

Fig.10. Preprocessing and FCM results of COR_IMG_01.
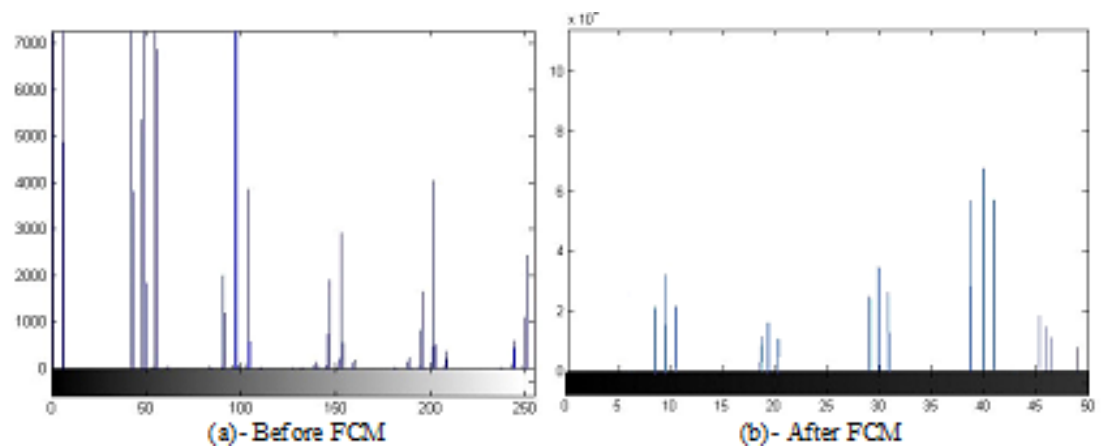

Fig.11. Histograms of COR_IMG_01

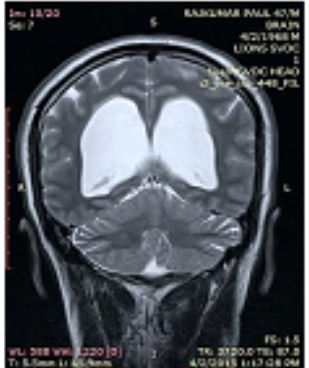

(a)

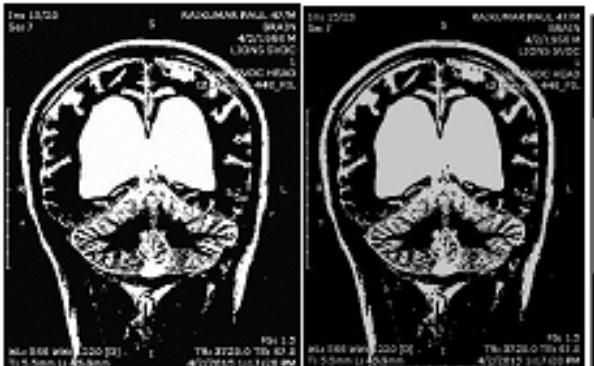

(b) (c)

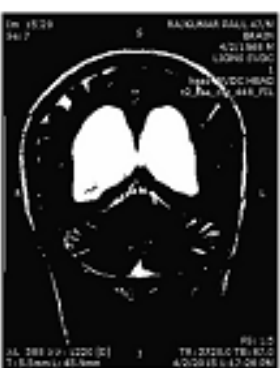

(d)

Fig. 12. Preprocessing and FCM results of COR_IMG_02.

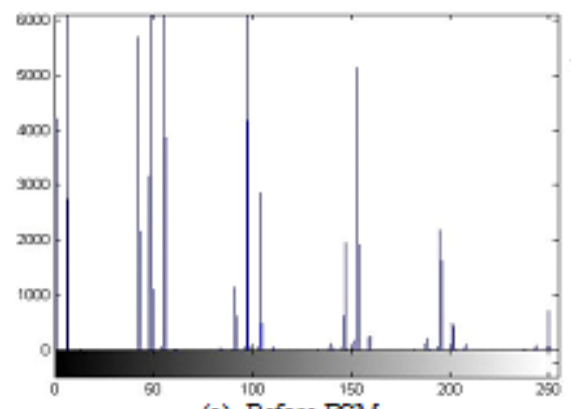

(a)- Before FCM

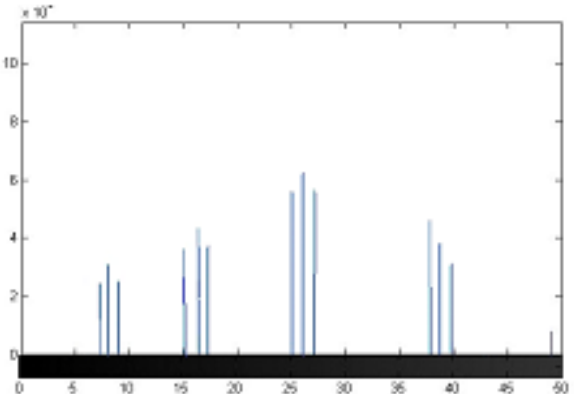

(b)- After FCM

Fig.13. Histograms of COR_IMG_02

In the coronal plane nearly 20 images are taken as input among that 3 image with their results are discussed here. The COR_IMG_02 is done with the preprocessing module and the traditional fuzzy c-means clustering algorithm is applied and the results are compared with the histogram values which are shown in the figures Fig. 12and Fig. 13 with the two cluster center values as $c 1=26.7629$ and $c 2=138.3620$. The COR_IMG_03 has undergone the same step of process as in the structured approach and produces the results as shown in the Fig. 14. and Fig. 15. With the cluster center values in the coordination of the histogram. The computation time for the three image of the coronal plane with their cluster center values obtained from the proposed approach is shown in the Table II. 


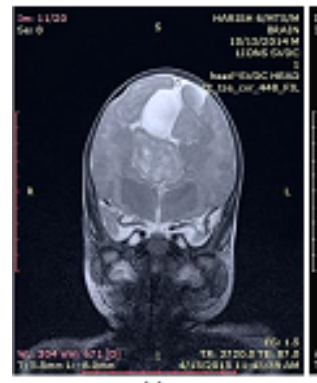

(a)

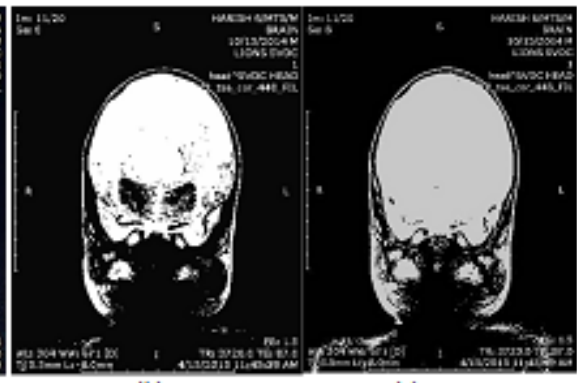

(b)

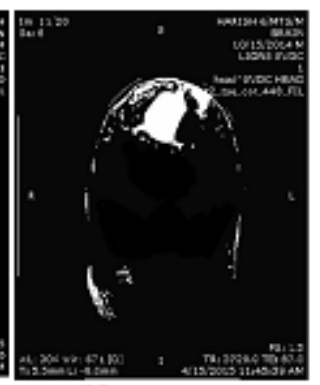

(d)

Fig. 14. Preprocessing and FCM resultsof COR_IMG_03.

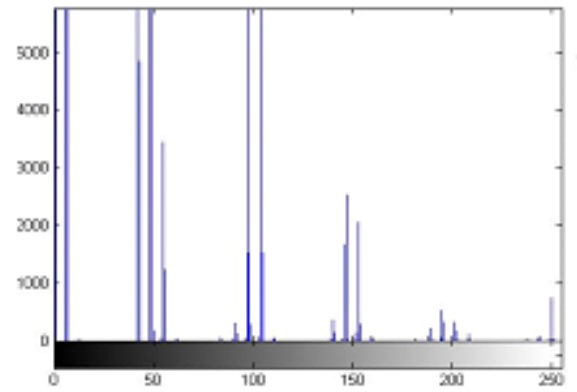

(a)- Before FCM

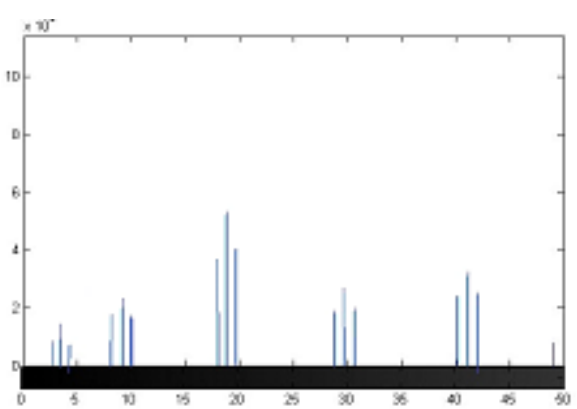

(b)- After FCM

Fig. 15. Histograms of COR_IMG_03

Table II: Computational time of the coronal plane

\begin{tabular}{|llll|}
\hline MRI IMAGE DATA SET & CLUSTER CENTER - C1 & CLUSTER CENTER - C2 & COMPUTATION TIME \\
COR_IMG_01 & 182.6475 & 40.0859 & 15.625922 \\
COR_IMG_02 & 138.3620 & 26.7629 & 18.734068 \\
COR_IMG_03 & 117.7723 & 19.6129 & 12.997683 \\
\hline
\end{tabular}

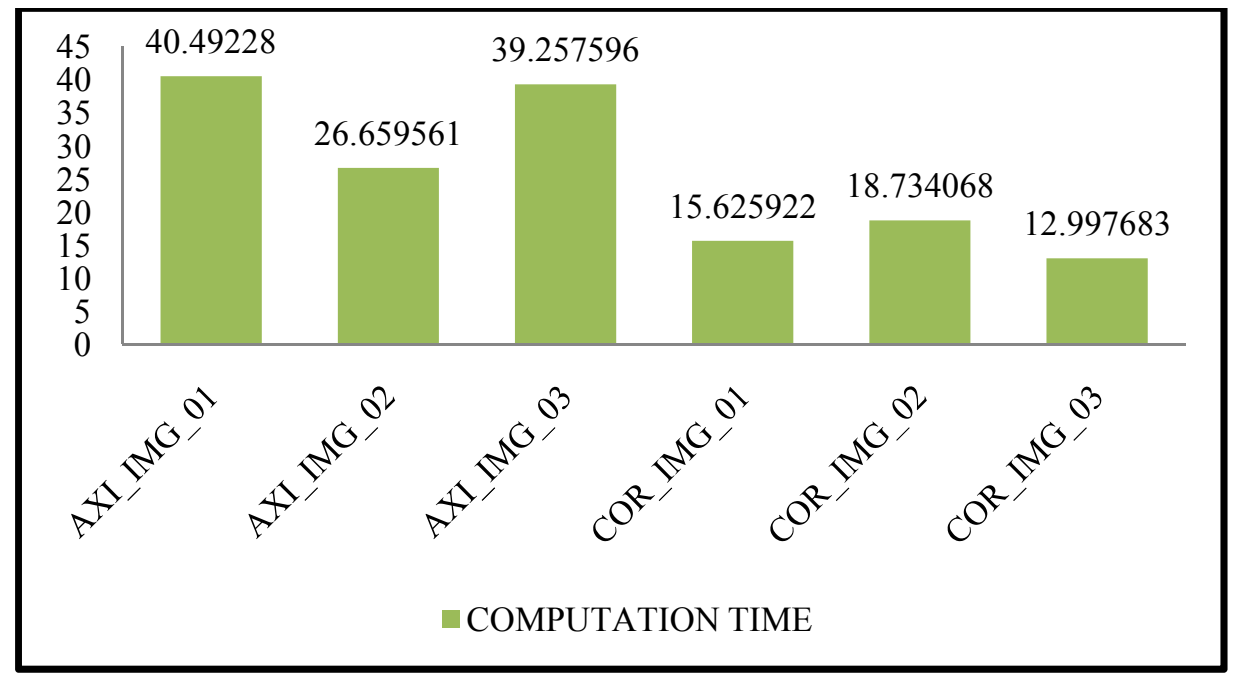

Fig. 16: Axial plane and coronal plane computational time comparison.

Fig. 16shows the comparative computational time of the axial and coronal plane for the three image data set. The computational time is calculated to discuss about the more efficiency of the FCM clustering algorithm in the real world over the axial and coronal plane. The computational time may differ based on the system specification. The time calculated is depends on the CPU evaluation time of only the fuzzy c means clustering algorithm. The axial plane has taken more time than the coronal plane. The elapsed time is calculated in seconds. In the real world, on diagnosis tumor to the large amount of patient the time consumption is an important criterion. On using the fuzzy c means for coronal plane it require only adequate time, so the FCM is well suitable for the coronal plane than the axial plane. 


\section{Conclusion}

The prediction of brain tumor is a critical problem in the medical field. The tumor affected region is separated from the MRI brain images by comparing the computational time of the axial and coronal plane and also finding the best fit of the FCM algorithm over the two planes. The structured approach discusses so far will help the physicians to detect the tumor affected region very easily. The preprocessing of images is carried out byGaussian filter method to remove the noisesfrom the images. After preprocessing, the traditional FCM is applied to segment the tumor affected region. The affected region is cross examined with the cluster center values of FCM. A physician can detect the tumor affected region very straightforwardly with the help of result of FCM algorithm. But, the FCM algorithm alone cannot diagnose the tumor for some type brain images. It helps the doctors or radiologists in finding the affected region perfectly to detect the tumor. The proposed approach is more suitable and robust for the coronal plane rather than the axial plane based on the computational time when comparing with the axial plane. The future work is to calculate the area of the tumor affected region by means of intensity based pixel values.

\section{References}

[1] Ahmed, M. N., Yamany, S. M., Farag, A. A., \& Moriarty, T. (1999). Bias field estimation and adaptive segmentation of MRI data using a modified fuzzy C-means algorithm. In Computer Vision and Pattern Recognition, 1999. IEEE Computer Society Conference on. (Vol. 1). IEEE.

[2] Ahmed, M. N., Yamany, S. M., Mohamed, N., Farag, A., \& Moriarty, T. (2002). A modified fuzzy c-means algorithm for bias field estimation and segmentation of MRI data. Medical Imaging, IEEE Transactions on, 21(3), 193-199.

[3] Alsmadi, M. K. (2015). MRI Brain Segmentation Using a Hybrid Artificial Bee Colony Algorithm with Fuzzy-C Mean Algorithm. Journal of Applied Sciences, 15(1), 100.

[4] Amini, L., Soltanian-Zadeh, H., Lucas, C., \&Gity, M. (2004). Automatic segmentation of thalamus from brain MRI integrating fuzzy clustering and dynamic contours. Biomedical Engineering, IEEE Transactions on, 51(5), 800-811.

[5] Chuang, K. S., Tzeng, H. L., Chen, S., Wu, J., \& Chen, T. J. (2006). Fuzzy c-means clustering with spatial information for image segmentation.computerized medical imaging and graphics, 30(1), 9-15.

[6] Clark, M. C., Hall, L. O., Goldgof, D. B., Velthuizen, R., Murtagh, F. R., \&Silbiger, M. S. (1998). Automatic tumor segmentation using knowledge-based techniques. Medical Imaging, IEEE Transactions on, 17(2), 187-201.

[7] Jose, A., Ravi, S., \&Sambath, M. (2014). Brain Tumor Segmentation Using K-Means Clustering And Fuzzy C-Means Algorithms And Its Area Calculation. Brain, 2(3).

[8] Kanade, P. B., \&Gumaste, P. P. (2015). Brain Tumor Detection Using MRI Images. Brain, 3(2).

[9] Kaur, M., Gupta, S., \&Bhushan, B. (2015). An Improved Adaptive Bilateral Filter to Remove Gaussian Noise from Color Images. International Journal of Signal Processing, Image Processing and Pattern Recognition, 8(3), 49-64.

[10] Khotanlou, H., Colliot, O., Atif, J., \& Bloch, I. (2009). 3D brain tumor segmentation in MRI using fuzzy classification, symmetry analysis and spatially constrained deformable models. Fuzzy Sets and Systems, 160(10), 1457-1473.

[11] Liew, A. W. C., \& Yan, H. (2003). An adaptive spatial fuzzy clustering algorithm for 3-D MR image segmentation. Medical Imaging, IEEE Transactions on, 22(9), 1063-1075.

[12] Liew, A. W. C., \& Yan, H. (2006). Current methods in the automatic tissue segmentation of 3D magnetic resonance brain images. Current Medical Imaging Reviews, 2(1), 91-103.

[13] Pham, D. L., \& Prince, J. L. (1999). An adaptive fuzzy C-means algorithm for image segmentation in the presence of intensity inhomogeneities. Pattern recognition letters, 20(1), 57-68.

[14] Tomasi, C., \&Manduchi, R. (1998, January). Bilateral filtering for gray and color images. In Computer Vision, 1998. Sixth International Conference on(pp. 839-846). IEEE.

[15] Vasuda, P., \&Satheesh, S. (1713). Improved Fuzzy C-Means algorithm for MR brain image segmentation. International Journal on Computer Science and Engineering, 2(5), 2010.

[16] Velmurugan, T. (2014). Performance based analysis between k-Means and Fuzzy C-Means clustering algorithms for connection oriented telecommunication data. Applied Soft Computing, 19, 134-146.

[17] Velmurugan,T. Performance Comparison between k-Means and Fuzzy C-Means Algorithms using Arbitrary Data Points. ." Wulfenia journal, Vol. 19, No. 8 (2012):234-241.

[18] Velmurugan, T., \&Santhanam, T. (2011). A comparative analysis between k-medoids and fuzzy c-means clustering algorithms for statistically distributed data points. Journal of Theoretical \& Applied Information Technology, 27(1).

[19] Wei, Y., \& Brown, H. K. (2015). A Novel Segmentation Approach For Brain Tumor in MRI.

[20] Zhao, M., Lin, H. Y., Yang, C. H., Hsu, C. Y., Pan, J. S., \& Lin, M. J. (2015). Automatic threshold level set model applied on MRI image segmentation of brain tissue. Appl. Math, 9(4), 1971-1980. 